\title{
Differences between hypothetical and experience-based value sets for EQ-5D used in Sweden: Implications for decision makers
}

Mattias Aronsson, Magnus Husberg, Almina Kalkan, Nathalie Eckard and Jenny Alwin

\author{
Linköping University Post Print
}

\section{Tweet}

N.B.: When citing this work, cite the original article.

Original Publication:

Mattias Aronsson, Magnus Husberg, Almina Kalkan, Nathalie Eckard and Jenny Alwin, Differences between hypothetical and experience-based value sets for EQ-5D used in Sweden: Implications for decision makers, 2015, Scandinavian Journal of Public Health, (43), 8, 848854.

http://dx.doi.org/10.1177/1403494815596910

Copyright: SAGE Publications (UK and US)

http://www.uk.sagepub.com/home.nav

Postprint available at: Linköping University Electronic Press

http://urn.kb.se/resolve?urn=urn:nbn:se:liu:diva-123133 
Differences between hypothetical and experience-based value sets for EQ-5D used in Sweden:

Implications for decision makers

Mattias Aronsson* M.Sc., Magnus Husberg B.Sc., Almina Kalkan Ph.D., Nathalie Eckard M.Sc., Jenny Alwin Ph.D.

Department of Medical and Health Sciences, Linkoping University, Linkoping, Sweden

Keywords: Health economics, Quality-Adjusted Life Years, EQ-5D, value set

Running title: Hypothetical and experience-based value sets

Word count: 2486

*Corresponding author:

Mattias Aronsson, M.Sc.

Linkoping University - Centre for Medical Technology Assessment

Department of Medical and Health Sciences

Linkoping University

SE-581 83 Linkoping, Sweden

Phone: +46 736219021

Fax: +46 101034995

Email: mattias.aronsson@liu.se 


\section{Abstract}

Aims

A number of value sets are available today for converting EQ-5D questionnaire responses to QALY-weights used in health economic evaluations. The aim of this study is to analyse the differences between the commonly used hypothetical UK value set and the newly introduced Swedish experience-based value set and to evaluate health economic implications of such differences on policy decisions.

\section{Methods}

Differences between the two value sets were studied using two methods: a comparison of health states and improvements as well as an empirical comparison. In the comparison of health states and improvements, the valuations of all EQ-5D states and all pure improvements were compared. In the empirical study, a database of 23,925 individuals was used to identify patient groups that could be affected by the implementation of the Swedish experience-based value set.

\section{Results}

The comparison of health states and possible improvements showed that only three health states were assigned a lower QALY-weight and most improvements were given smaller absolute values if the experience-based value set was used. The empirical comparison showed that severe conditions were assigned higher values when using the experience-based value set.

\section{Conclusions}

The Swedish experience-based value set seems to render a higher estimated level of HRQoL in virtually all health conditions compared to the hypothetical UK value set. In extension, HRQoL enhancing interventions are likely to be given higher priority in decision making situations where hypothetical values are used to construct QALY-weights. In situations where experience-based QALY-weights are used, life-prolonging interventions would be prioritised.

Keywords: Economic evaluations, quality-Adjusted Life Years, EQ-5D. 


\section{Introduction}

A growing number of health economic evaluations are being conducted within health care to serve as the basis for resource allocation decisions and priority setting. These evaluations are focused on the costs and effects of interventions under investigation. The effects are often expressed as quality-adjusted life years (QALYs), which is a standardised outcome measure obtained by multiplying the number of life years in a given health state with the health-related quality of life (HRQoL) in that state. The HRQoL in a state is, therefore, often expressed as a QALY-weight.

QALY-weights are traditionally calculated from the responses of individuals in standardised instruments. A widely used generic instrument for this purpose is the EQ-5D questionnaire, which was originally developed by the EuroQol Group and published in 1990 [1]. The instrument contains five dimensions (5D) as follows: mobility, self-care, usual activities, pain/discomfort, and anxiety/depression. Traditionally, in this instrument, the respondents have to answer five questions based on a three-point scale: no problems, moderate problems, and severe problems. Each combination of the responses represents one specific health state, resulting in 243 (3 $\wedge$ 5) different health states. The health states can be used for cost-effectiveness analyses by assigning a specific QALY-weight to each health state based on an index (where 1 represents full health and 0 represents death). In 1997, the first value set for conversion of the EQ-5D questionnaire into QALY-weights was published by Dolan [2], in which British citizens had evaluated hypothetical health states through the time trade-off (TTO) method.

Since 1997, value sets for many countries have been developed [3-12]. Most value sets have been developed based on hypothetical evaluations of the health states by representatives from the society. However, a Swedish value set [12] based on individuals who evaluated their own health state was published in 2013, and has since then received growing attention in Swedish healthcare policy. The hypothetical UK value set published by Dolan is the most commonly used value set in Sweden as well as in many other European countries. This value set is also advocated by authorities such as the National Institute of Health and Clinical Excellence (NICE) [13] with the main argument being that health care is, more or less, collectively financed. However, several authors have suggested that experience-based values should be used instead since individuals who experience a certain health state have the best insight regarding the HRQoL of their health state [14-16]. Despite major differences in terms of perspective and also methodology (presented in the next section) between the hypothetical UK value set [2] and the new Swedish experience-based value set [12], the experience-based values are now starting to be used by policymakers in Sweden [17]. Since both these value sets are used in 
Sweden today, it is important to study and understand the potential differences they generate in terms of results in cost-effectiveness analyses and its impact on decision making.

Therefore, in this study, we aim to analyse the differences between the hypothetical UK value set and the Swedish experience-based value set in terms of QALY-weights derived from the EQ-5D instrument. Furthermore, we aim to explore health economic implications of the use of the Swedish experience-based value set for policy decisions in health care.

\section{Method and Materials}

\section{Hypothetical and experience-based value sets}

In order to convert the EQ-5D health states to QALY-weights, we have used both the hypothetical UK value set from Dolan [2] and the Swedish experience-based value set from Burström et al. [12] in our study. Dolan [2] based his study on the responses of 2997 British individuals evaluating 42 different hypothetical EQ5D-health states (each respondent answered questions about 13 different health states) using time-trade-off (TTO) method. Concisely, in this method, the individuals were asked to answer the number of life years in a given state they would be willing to trade for life in full health. QALY-weights for 42 states were created based on the responses. Using statistical models (generalized least squares), weights could be estimated also for those states that were not included in the trial. Burström's et al. [12] experience-based value set was based on two merged surveys conducted in Sweden: one in the Scania region in 2004 and the other in Stockholm in 2006. In their study, 45,477 individuals answered the EQ-5D questionnaires and TTO-questions about their health states. A total of 148 different states were reported and valued in their study, and seven different statistical models to convert EQ-5D states to QALY-weights were presented. We have consistently used Model 4 (a model based on ordinary least squares method) in our current study because its structure best matched the frequently-used hypothetical UK value set by Dolan [2].This model was also recommend by the by the authors [12]. A conceptual difference between the value sets is, as shown in Table 1, that the best possible health state (11111) is valued to be 1 when using hypothetical values and 0.97 when using experience-based value set. Another important remark is that it is not possible to assign health states worse than death in the Swedish experiencebased value set; thus, no health states were assigned negative values. Negative values are however possible to get when using the hypothetical UK value set. 


\section{Comparison of health states and improvements}

To study the difference between the two value sets, we compared the valuation of all 243 different EQ-5D states. In addition, we also compared how the two value sets valued all (7533) pure improvements, i.e. improvements in which at least one dimension improved without any deterioration in another dimension.

The differences between the two sets were then validated for country specific differences using published value sets from the UK, Germany, Poland, Denmark, France, Netherlands, Spain, USA, Japan and South Korea $[2,4-10,18,19]$. (The results of the validation analyses are available as supplementary material online.)

\section{Empirical comparison}

A database of 23,925 individuals was used to study and identify specific patient groups that could be affected by implementing the experience-based value set rather than the hypothetical value set. The individuals in the database had responded to a survey conducted during a week in the outpatient care in Östergötland County, Sweden, in the years 2000 and 2002. A total of 22,556 (94.3\%) individuals answered all of the five EQ5D questions, while 21,817 (91.2\%) responded both to the EQ-5D questions and the question regarding the clinic they had visited. The information about the clinics that were visited enabled us to roughly group the patients into twelve different disease categories. A detailed description of data collection is presented in other studies [20, 21].

\section{Statistical Analysis}

All statistical data were handled and analysed in SPSS 22 (SPSS Inc., Chicago, IL). Calculations were carried out in MathWorks MATLAB (MathWorks, Natick, MA) and Microsoft Excel 2010 (Microsoft, Redwood, WA).

\section{Results}

\section{Comparison of health states and improvements}

The traditionally used hypothetical UK value set [2] assigned most health states lower values compared to the experience-based Swedish value set [12] (Figure 1). Only three (1.2\%) health states had a lower QALYweight using the experience-based value set (11111, 11211 and 21211). As shown in Figure 1, approximately one third of the health states have negative values in the UK value set by Dolan [2]. The jagged appearance of 
the experience-based valuation is because the values were ranked from highest-to-lowest by the hypothetical value set and the value sets in relation to each other did not rank health states in the same order. Furthermore, no major differences were found in the result if hypothetical value sets from other countries were used in comparison with the Swedish experience-based value set (validation analyses are available as supplementary material online).

The use of the experience-based weights resulted in a smaller improvement compared to the hypothetical weights in $95.4 \%(7,189$ of 7,533$)$ of all possible pure improvements (Figure 2). A health improvement valued to one QALY with the hypothetical UK value set was, as shown by the trend line in Figure 2, in average valued to approximately 0.38 QALYs with the Swedish experience-based value set.

\section{Empirical comparison}

Table 2 shows how the choice of value set affects QALY-weights for different patient characteristics (gender and age) and patient populations. In general, as specified in Table 2, the value set with experience-based values provides higher QALY-weights. The differences seems to increase with age and are highest in neurological and psychiatric conditions.

\section{Discussion}

Our study showed that the choice between the hypothetical value set and the experience-based value set may be fundamental for the estimation of QALY-weights. This will, in extension, have an impact on the results of cost-effectiveness analyses and policy making.

To illustrate the potential impact for health economic decisions we use basic examples of two extreme scenarios. In the first example, an otherwise healthy patient suffering from severe pain (EQ-5D state: 11131) becomes free from pain (EQ-5D state: 11111 ) during a year to a cost of $€ 5,000$. The traditionally used hypothetical UK value set would estimate the QALY gain of the intervention to 0.74 QALYs (an increase from 0.26 to 1 ), whereas the QALY gain with experience-based value set would be 0.13 (an increase from 0.84 to 0.97). This implies an incremental cost of $€ 6,759$ per gained QALY using the hypothetical value set, while the incremental cost when using the Swedish experience-based value is set is six times higher (€ 38,462 per gained QALY). However, if the same patient was treated with an intervention that instead prolonged the patient's life by one year to a cost of the $€ 5,000$ it would imply cost of approximately $€ 19,231$ per QALY gained using the hypothetical value set as compared to $€ 5,952$ per QALY gained when using the experience-based values. As 
QALY-weights are being widely used in decision making regarding health care technologies in Sweden, the choice between these two value sets are likely to affect results and thereby also decisions. In decision making situation where hypothetical values are used to construct QALY-weights HRQoL enhancing interventions would be given higher priority. On the other hand, in situations where experience-based QALY-weights are used, life-prolonging interventions would be prioritised.

We did not identify any apparent differences in the estimated HRQoL in different patient populations. However, Table 1 shows that the largest difference between the two value sets were found in the dimension pain/discomfort while there was only a minor difference in the dimension anxiety/depression. Hence, the choice of value set would affect the result of cost-effectiveness analyses more if the dimension pain/discomfort is affected by an intervention compared to the dimension anxiety/depression. This could, if the new Swedish value set is starting to be more widely used, imply a re-allocation of resources between different patient populations.

The differences between and potential explanatory parameters of how patients and the general population give value to health states have been previously analysed [22, 23].The choice between hypothetical and experience-based value sets is extensively debated as both methods have advantages as well as disadvantages. One viewpoint brought forward for using hypothetical values is that interventions may affect the society; the health care is, more or less, collectively financed and everyone can potentially become a patient, therefore societal preferences should be used in the valuation. The main reason for using experience-based weights lies with the argument that individuals affected with a certain condition are most likely to have the best insight about the appropriate level of HRQoL and thus, the QALY-weight, see e.g. [12, 15, 16, 23-25]. The aim of this analysis is not to advocate either of the two perspectives, but instead illustrate the implications of the choice between value sets.

The two value sets studied in this analysis are based on different types of populations and cultures (UK and Sweden). Comparing value sets from these populations may be considered a limitation as studies have shown that differences between value sets partially can depend on country-specific preferences [19, 26-28]. However, our results were consistent even when hypothetical value sets from other European countries (UK, Germany, Poland, Denmark, France, Netherlands and Spain) were compared with the Swedish experienced-based value set. The results were also fairly consistent if the Swedish value set was compared with hypothetical value sets from non-European countries such as USA, Japan and South Korea. Country-specific differences exist, but are likely minor compared to the differences between experience-based and hypothetical value set (a short 
presentation of the validation results is available as supplementary material online). As presented earlier in this paper, in addition to differences in perspective, there are also methodological differences between the Swedish experience-based and the hypothetical UK value sets. For instance, in the value set by Burström et al. [12] all subjects answered TTO-questions in population surveys while the TTO-questions were answered in interviews in the value set published by Dolan [2]. The authors of the Swedish experience-based value set also discuss inconsistencies in their data material [12]. Important issues are how the TTO-questions have been described to and valued by the respondents. Burström et al. [12] point out problems of age e.g. older respondents is prone to misunderstand survey questions on the dimension of self-care. They also show that gender is an important health indicator for e.g. the dimension of anxiety/ depression. However, the sample size in the Swedish study provides a strong foundation for the results. It is also important to point out that the two value sets are measuring different parameters (hypothetical respectively experienced HRQoL) which make direct comparisons difficult.

Regardless of which of these two value sets that are considered adequate to use, the authors of the present study believe that it is important to show and analyse the differences. Strategic behaviour may otherwise occur if authorities and decision makers are not aware of the differences and thereby allow e.g. companies and individuals to get desired results by choosing the value set that provide the lowest cost per QALY gained.

Further research is needed to analyse how the choice of value set could affect the threshold for when a treatment should be considered cost-effective. A treatment previously considered cost-effective could be regarded as ineffective if the experience-based value set is implemented and thereby potentially cause a reallocation of resources (Figure 3). This could also raise questions concerning consistency in health care policy making.

\section{Conclusions}

The Swedish experience-based QALY-weights derived from EQ-5D would increase the estimated level of HRQoL in virtually all health conditions compared to the commonly used hypothetical UK value set. This implies that HRQoL enhancing interventions would be given higher priority in decision making situations where hypothetical values are used to construct QALY-weights. On the other hand, in situations where the experiencebased QALY-weights are used, life-prolonging interventions would be prioritised. It is, therefore, important that decision makers are aware of the implications that the choice of value set entails. 
Conflict of Interest: This study was supported by unrestricted grants from the County Council of Östergötland. The authors declare no conflict of interest.

Ethics: This study was approved by the Regional Ethics Committee in Linköping, Sweden (Dnr 2013/243-31). 


\section{References}

1. EuroQol--a new facility for the measurement of health-related quality of life. The EuroQol Group. Health Policy 1990; 16: 199-208.

2. $\quad$ Dolan P. Modeling valuations for EuroQol health states. Med Care 1997; 35: 1095-108.

3. Yusof FA, Goh A, Azmi S. Estimating an EQ-5D value-set for Malaysia using time trade-off and visual analogue scale methods. Value Health 2012; 15: S85-90.

4. Wittrup-Jensen KU, Lauridsen J, Gudex C, et al. Generation of a Danish TTO value-set for EQ-5D health states. Scand J Public Healt 2009; 37: 459-66.

5. Tsuchiya A, Ikeda S, Ikegami N, et al. Estimating an EQ-5D population value-set: the case of Japan. Health Econ 2002; 11: 341-53.

6. Shaw JW, Johnson JA, Coons SJ. US valuation of the EQ-5D health states: development and testing of the D1 valuation model. Med Care 2005; 43: 203-20.

7. Lamers LM, McDonnell J, Stalmeier PF, et al. The Dutch tariff: results and arguments for an effective design for national EQ-5D valuation studies. Health Econ 2006; 15: 1121-32.

8. Jo MW, Yun SC, Lee SI. Estimating quality weights for EQ-5D health states with the time trade-off method in South Korea. Value Health 2008; 11: 1186-9.

9. Greiner W, Claes C, Busschbach JJ, et al. Validating the EQ-5D with time trade off for the German population. Eur J Health Econ 2005; 6: 124-30.

10. Golicki D, Jakubczyk M, Niewada M, et al. Valuation of EQ-5D health states in Poland: first TTO-based social value-set in Central and Eastern Europe. Value Health 2010; 13: 289-97.

11. Augustovski FA, Irazola VE, Velazquez AP, et al. Argentine valuation of the EQ-5D health states. Value Health 2009; 12: 587-96.

12. Burstrom K, Sun S, Gerdtham UG, et al. Swedish experience-based value-sets for EQ-5D health states. Qual Life Res 2013.

13. NICE. Guide to the methods of technology appraisal. London: National Institute for Health and Clinical Excellence. London, 2008.

14. Dolan P. NICE should value real experiences over hypothetical opinions. Nature 2009; 462: 35.

15. Leidl R, Reitmeir P. A value set for the EQ-5D based on experienced health states: development and testing for the German population. PharmacoEconomics 2011; 29: 521-34.

16. Dolan P, Kahneman D. Interpretations of utility and their implications for the valuation of health. Econ J. 2008; 118: 215-34.

17. The Dental and Pharmaceutical Benefits Agency (TLV). Stivarga kommer inte att ingå i högkostnadsskyddet. [Stivarga will not be reimbursed]. TLV decision: 3527/2013, Stockholm, 2014.

18. Chevalier J, de Pouvourville G. Valuing EQ-5D using time trade-off in France. Eur J Health Econ 2013; 14: 57-66.

19. Badia X, Roset M, Herdman M, et al. A comparison of United Kingdom and Spanish general population time trade-off values for EQ-5D health states. Med Decis Making 2001; 21: 7-16.

20. Rahmqvist M, Bara AC. Patient characteristics and quality dimensions related to patient satisfaction. Int $J$ Qual Health C 2010; 22: 86-92.

21. Rahmqvist M, Bara AC. Patients retrieving additional information via the Internet: a trend analysis in a Swedish population, 2000-05. Scand J Public Healt 2007; 35: 533-9.

22. Stiggelbout AM, de Vogel-Voogt E. Health state utilities: a framework for studying the gap between the imagined and the real. Value Health 2008; 11: 76-87.

23. Ubel PA, Loewenstein G, Jepson C. Whose quality of life? A commentary exploring discrepancies between health state evaluations of patients and the general public. Qual Life Res 2003; 12: 599-607.

24. De Wit GA, Busschbach JJ, De Charro FT. Sensitivity and perspective in the valuation of health status: whose values count? Health Econ 2000; 9: 109-26.

25. Gold MR, Siegel JE, Russell LB, et al. Cost-Effectiveness in Health and Medicine. Oxford University Press, USA, 1996.

26. Bailey H, Kind P. Preliminary findings of an investigation into the relationship between national culture and EQ-5D value-sets. Qual Life Res 2010; 19: 1145-54.

27. Knies S, Evers SM, Candel MJ, et al. Utilities of the EQ-5D: transferable or not? PharmacoEconomics 2009; 27: 767-79.

28. Johnson JA, Luo N, Shaw JW, et al. Valuations of EQ-5D health states: are the United States and United Kingdom different? Med Care 2005; 43: 221-8. 


\section{Figure legends}

Figure 1.The relationship between the Swedish experience-based [12] and the hypothetical UK value set [2].

The figure shows the valuation of all 243 different EQ-5D states ranked from highest-to-lowest by the hypothetical value set. The labels on the X-axis show only a sample of possible health states.

Figure 2. All possible pure health improvements valued by the experience-based value set in comparison with the hypothetical valuation. Values to the right of the red line represent health improvements that are valued higher with the hypothetical value set. 


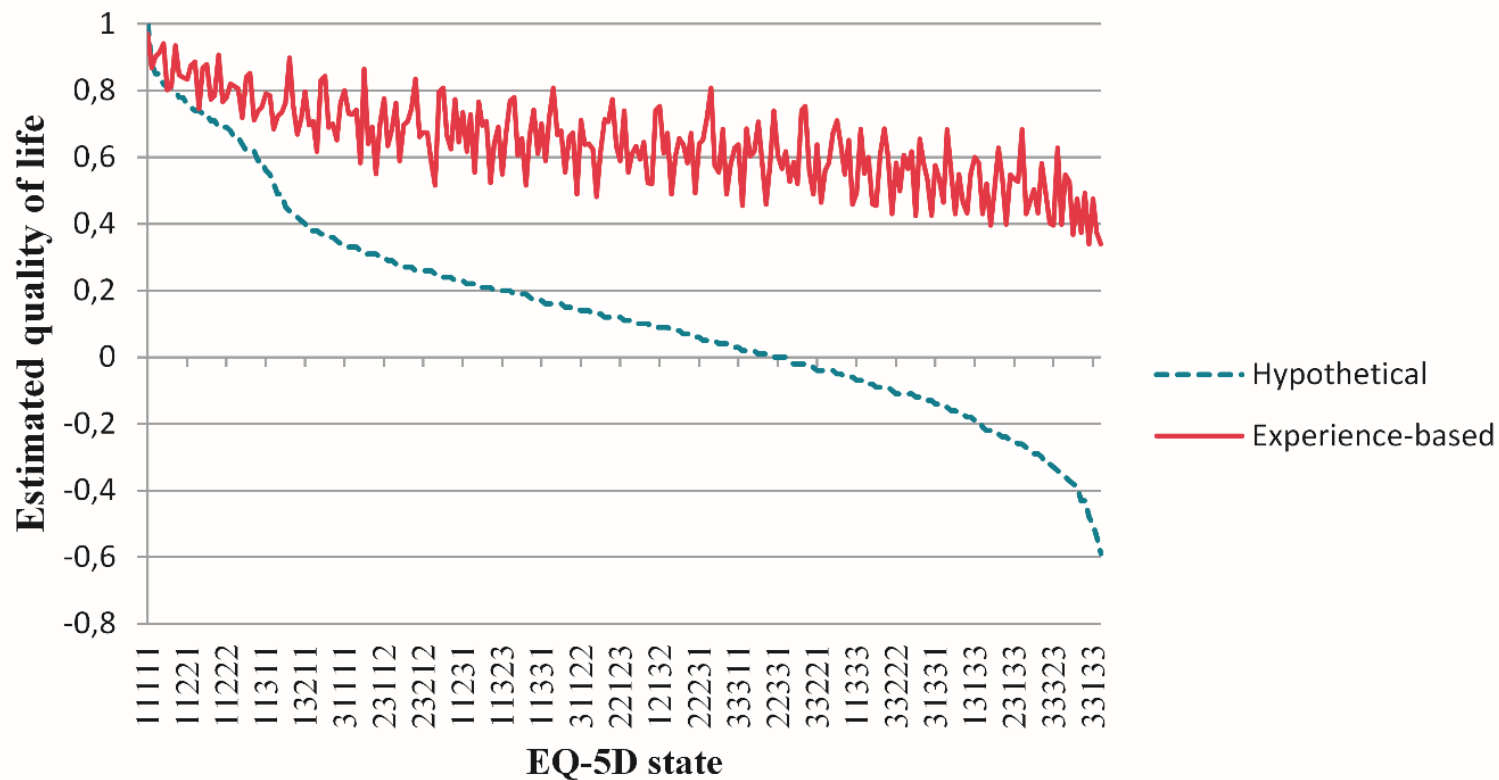




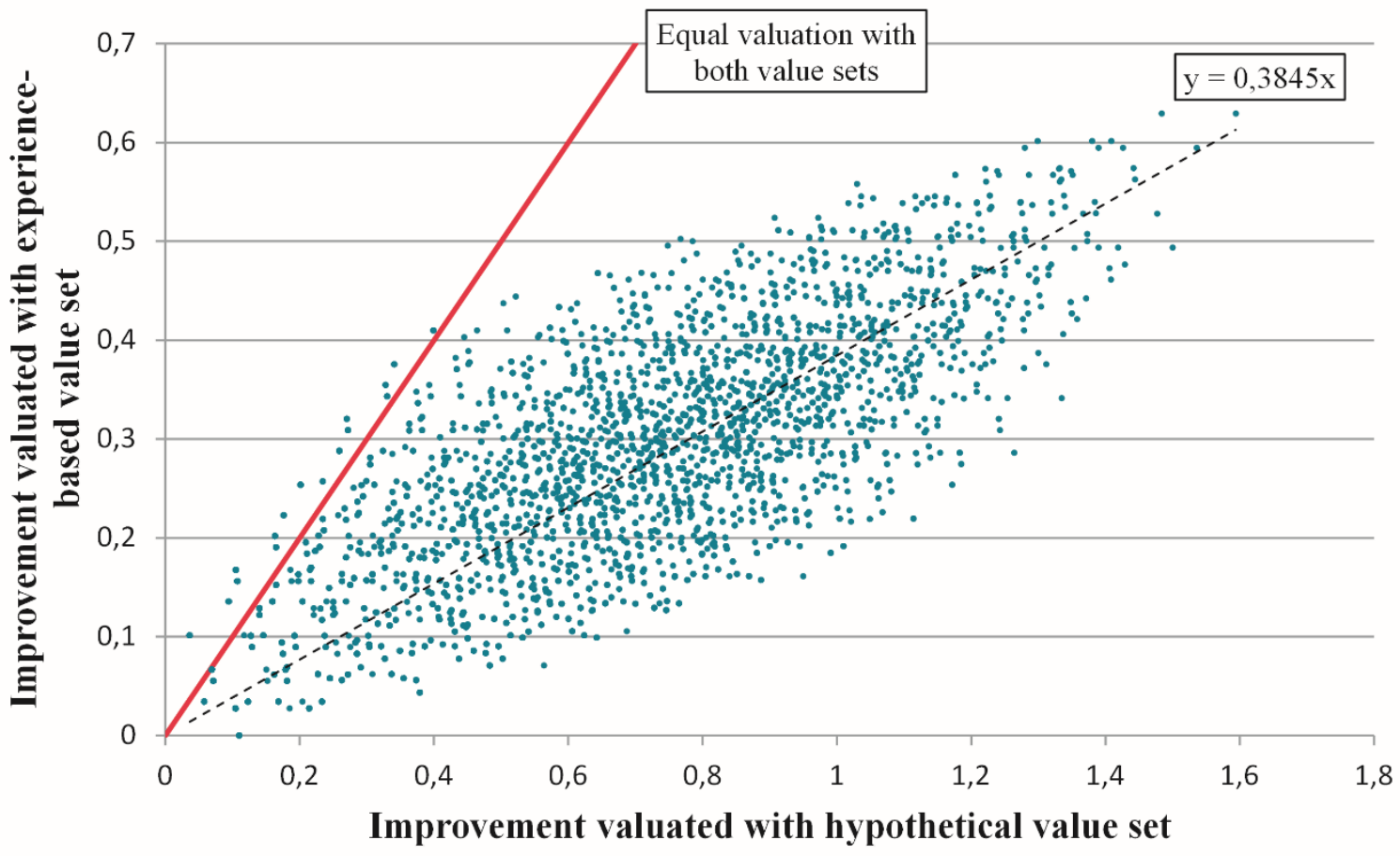

